\title{
Efficient Ultra-broadband Optical Parametric Generation with Picojoule Pulse Energies
}

\author{
Marc Jankowski $^{1,2, *}$, Nayara Jornod ${ }^{1, *}$, Carsten Langrock ${ }^{1}$, Boris Desiatov ${ }^{3}$, Alireza \\ Marandi $^{4}$, Marko Lončar ${ }^{3}$, Martin M. Fejer ${ }^{1}$ \\ ${ }^{1}$ Edward L. Ginzton Laboratory, Stanford University, Stanford, California 94305, USA \\ ${ }^{2}$ NTT Research Inc. Physics and Informatics Labs, 940 Stewart Drive, Sunnyvale, California \\ ${ }^{3}$ John A. Paulson School of Engineering and Applied Sciences, Harvard University, Cambridge, Massachusetts \\ 02138, USA \\ ${ }^{4}$ Department of Electrical Engineering, California Institute of Technology, Pasadena, California 91125, USA \\ ${ }^{*}$ These authors contributed equally \\ marcjank@stanford.edu
}

\begin{abstract}
We demonstrate high-gain optical parametric generation in dispersionengineered PPLN nanowaveguides with picojoules of pump pulse energy. When driven with $>10 \mathrm{pJ}$, the generated signal exceeds $10 \%$ conversion efficiency and broadens to span an octave of bandwidth. (C) 2021 The Author(s)
\end{abstract}

\section{Introduction}

Parametric down conversion enables the generation and amplification of long-wavelength signals from a shortwavelength pump. In the absence of a coherent seed, this process is used as a source of single photons or squeezed light. When seeded with a coherent signal or embedded in a resonator, parametric down conversion is used to produce broadband coherent sources for spectroscopy at otherwise difficult to access wavelengths. Parametric sources benefit from an inherently broad gain, limited only by the dispersion and transparency of the material. However the quadratic nonlinearities used for parametric down-conversion are weak, and therefore require high intensities and long interaction lengths. The energy required to achieve sufficient gain for efficient operation has therefore been limited by interplay of spatial and temporal walk-offs as well as dispersion and diffraction in the nonlinear device. In the case of optical parametric generation (OPG), where vacuum fluctuations are amplified to macroscopic intensities, state-of-the-art devices based on quasi-phase-matched (QPM) interactions in diffused waveguides operate with nanojoules of pulse energy, limited both by the spatial confinement of the interacting modes and by the effective interaction lengths for short pulses (Fig. 1a) [1].

a)

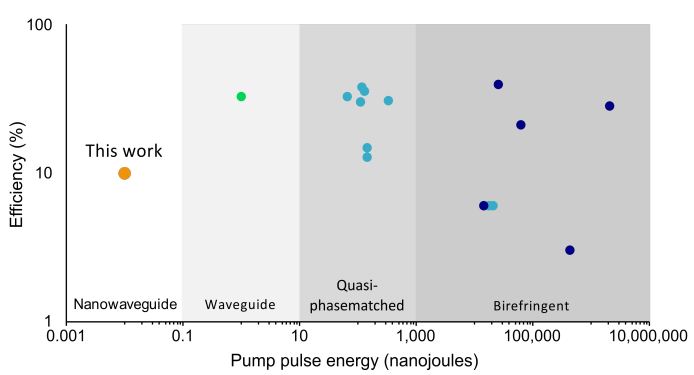

b)

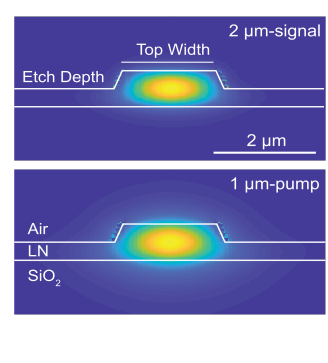

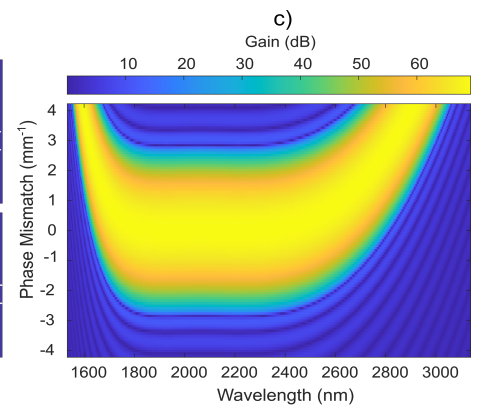

Fig. 1. a) Overview of the efficiency of OPGs as a function of input pulse energy. b) Waveguide cross-section and the normalized electric field of simulated $\mathrm{TE}_{00}$ pump (lower) and it generated signal (upper). c) Simulated OPG bandwidth for a pump pulse energy of $2 \mathrm{pJ}$.

In this work we present the efficient OPG with only picojoules of pump energy. This drastic reduction in required pulse energy is made possible by recent progress in periodically poled thin film lithium niobate (TFLN) nanowaveguides $[2,3]$. This new technology provides sub-wavelength spatial confinement, which increases the fields intensities of the interacting modes by an order of magnitude. Furthermore, the geometry dependence of the dispersion of the interacting waves opens up the possibility of engineering the interaction lengths of short pulses by eliminating group velocity mismatch (GVM) and group velocity dispersion (GVD) of the interacting harmonics. In this case, we eliminate both GVM and the GVD of the fundamental, thereby enabling a 6-mm-long waveguide to be driven with an 80-fs pump pulse. When driven with pulse energies in excess of $10 \mathrm{pJ}$, these devices achieve octave spanning OPG with an efficiency above $10 \%$. We observe a saturated gain of $83 \mathrm{~dB}$ with only $18 \mathrm{pJ}$ of pump energy. 


\section{Results}

The gain bandwidth available for OPG near degeneracy is dominated by the GVD of the generated signal, and pump bandwidth available to contribute to OPG is limited by the GVM between the pump and the generated signal. When both GVD and GVM are set close to zero, the interacting waves co-propagate along the length of the device while keeping high temporal confinement, enabling high gain with large bandwidth. In this experiment we use a 6-mm long TFLN nanowaveguide with a film thickness of $700 \mathrm{~nm}$, a top width of $1850 \mathrm{~nm}$ and an etch depth of $340 \mathrm{~nm}$ (Fig. 1b). This geometry enables a GVD of $k_{\omega}^{\prime \prime} \sim 6 \mathrm{fs}^{2} / \mathrm{mm}$ and a GVM of $\Delta k^{\prime} \sim 5 \mathrm{fs} / \mathrm{mm}$. Figure 1c shows the calculated gain available from this device with a pump of $80 \mathrm{fs}$ centered at $1045 \mathrm{~nm}$ and a pulse energy of $2 \mathrm{pJ}$. When the phase-mismatch between the pump and signal is close to zero, high gain is expected over nearly a micron of bandwidth $(\sim 1700-2600 \mathrm{~nm})$. We note here that the gain bandwidth can be further enhanced with a small phase-mismatch, and we therefore operate these waveguides with $\Delta k \sim 2 \mathrm{~mm}^{-1}$ to achieve broad gain from $1600-2800 \mathrm{~nm}$.

Figure 2a shows the setup used to characterize the waveguides. We pumped the device with 80 -fs pulses from a modelocked laser emitting at $1047 \mathrm{~nm}$ and with a repetition frequency of $100 \mathrm{MHz}$. Using a lock-in amplifier, we could measure a generated signal in the $2-\mu \mathrm{m}$ range with only 60 femtojoules of pump energy. For pump energies below $5 \mathrm{pJ}$ the parametric generation remains in the undepleted gain regime, as depicted in the inset of Figure $2 \mathrm{~b}$. In this case the output power follows the predicted theoretical gain and the optical spectrum (Fig. 2c) agrees well with the expected bandwidth from Fig. 1c. When driving the waveguides with pulse energies above 10 picojoules, the process enters a saturated gain regime. The pump and the generated signal broadens to merge and span an octave of bandwidth. The conversion efficiency and the pump depletion are presented in Figure $2 \mathrm{~b}$. With $18 \mathrm{pJ}$ of pump energy we could generate $200 \mu \mathrm{W}(2 \mathrm{pJ})$ of signal, which corresponds to a conversion efficiency of $11 \%$ and a saturated gain of $83 \mathrm{~dB}$. We note here that the pump depletion ( $>60 \%)$ exceeds the signal conversion efficiency. We attribute this energy loss to parasitic second-harmonic generation (SHG) of the pump. Further investigation on the SHG losses and broadening mechanisms in play in the experiment are done with simulations.

a)

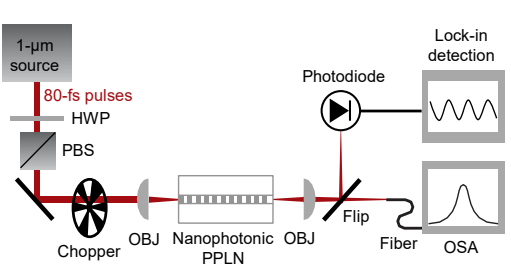

b)

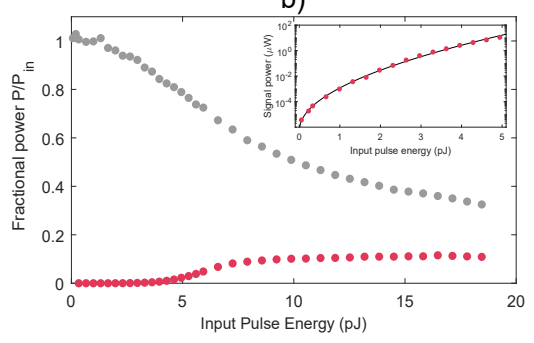

c)

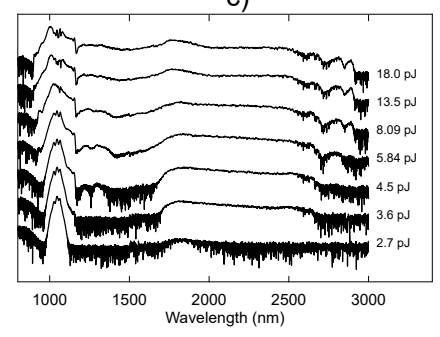

Fig. 2. a) Experimental setup. HWP, half-wave plate; PBS, polarizing beamsplitter; OBJ, reflective objective lens; OSA, optical spectrum analyzer. b) Conversion efficiency (red) and pump depletion (grey). Inset: Semilog scale of the generated power before saturation. c) Selection of measured power spectral density. Adjacent traces are shifted by $30 \mathrm{~dB}$ to facilitate readability.

\section{Conclusion}

We have experimentally demonstrated efficient optical parametric generation of an ultrabroadband signal with only $18 \mathrm{pJ}$ of pump energy in dispersion-engineered TFLN nanowaveguides. Our devices enable parametric down conversion with pulse energies that are orders of magnitude lower than previous demonstrations, enabled by careful geometric design that suppress both temporal walk-off and pulse spreading. We expect these results to enable a new class of chip-scale parametric devices that operate with orders of magnitude less power than previous generation technologies.

\section{References}

1. X. Xie et al.,"'Picojoule threshold, picosecond optical parametric generation in reverse proton-exchanged lithium niobate waveguides," J. Opt. Soc. Am. B 21, 1397 (2004).

2. C. Wang et al.,"Ultrahigh-efficiency wavelength conversion in nanophotonic periodically poled lithium niobate waveguides," Optica 5, 1438 (2018).

3. M. Jankowski et al.,"Ultrabroadband nonlinear optics in nanophotonic periodically poled lithium niobate waveguides," Optica 7, 40 (2020). 